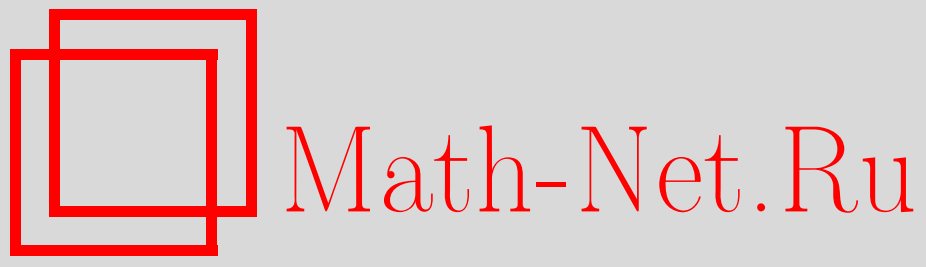

А. М. Гомилко, О факторизации оператор-функций в гильбертовом пространстве, Функи. анализ и его прил., 2003, том 37, выпуск 1, 19-24

DOI: https://doi.org/10.4213/faa133

Использование Общероссийского математического портала MathNet.Ru подразумевает, что вы прочитали и согласны с пользовательским соглашением

http://www . mathnet.ru/rus/agreement

Параметры загрузки:

IP : 34.229 .45 .116

26 апреля 2023 г., 15:53:18

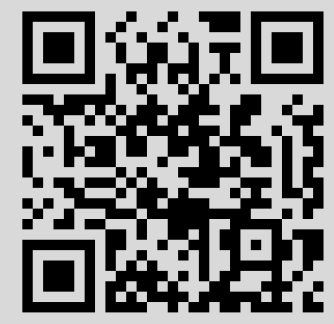


Функциональный анализ и его приложения

2003, т. 37, вып. 1, с. 19-24

УДК 517.9

\title{
О факторизации оператор-функций \\ в гильбертовом пространстве
}

\author{
(C) 2003. А. М. Гомилко
}

1. Введение. Пусть $H$ - гильбертово пространство со скалярным произведением $(\cdot, \cdot)$ и нормой $\|\cdot\|$, а $L=L(H)$ - алгебра всех линейных ограниченных операторов, действующих в $H$. Через $\sigma(A)$ обозначаем спектр оператора $A \in L(H), A^{*}$ - сопряженный к $A$ оператор, $I$ - единичный оператор и $R(A, \lambda)=(A-\lambda I)^{-1}, \lambda \notin \sigma(A),-$ резольвента оператора $A$. Для оператора $A \in L$ через $W(A)$ обозначаем его числовой образ: $W(A)=\{(A f, f):\|f\|=1\}$.

Всюду далее $\Gamma \subset \mathbb{C}-$ окружность единичного радиуса с началом в $\lambda=0$, которая разбивает комплексную плоскость на области $G^{+}=\{\lambda:|\lambda|<1\}$ и $G^{-}=\{\lambda:|\lambda|>1\}$. Пусть $C(\Gamma, L)\left(H_{\alpha}(\Gamma, L)\right)$ - алгебра всех непрерывных (удовлетворяющих условию Гёльдера с показателем $\alpha$ ) оператор-функций на $Г$ со значениями в $L$. Через $H_{\alpha}^{+}(\Gamma, L)\left(H_{\alpha}^{-}(\Gamma, L)\right)$ обозначаем подалгебру алгебры $H_{\alpha}(\Gamma, L)$, состоящую из оператор-функций, которые допускают голоморфные продолжения в $G^{+}\left(G^{-}\right)$. Через $\operatorname{ind}_{\Gamma} a(\zeta)$ будем обозначать индекс функции $a(\zeta) \in C(\Gamma)(a(\zeta) \neq 0, \zeta \in \Gamma)$.

Данная заметка посвящена обобщению следующего результата А. С. Маркуса и В. И. Мацаева [1]: если оператор-функция $A(\zeta)$ принадлежит $H_{\alpha}^{+}(\Gamma, L), \alpha \in$ $(0,1)$, причем

$$
\inf _{\zeta \in \Gamma,\|f\|=1}|(A(\zeta) f, f)|>0
$$

и функция $(A(\lambda) f, f)(f \neq 0)$ имеет в круге $G^{+}$ровно $k$ корней, то имеет место факторизация

$$
A(\lambda)=A_{+}(\lambda) P_{k}(\lambda), \quad P_{k}(\lambda)=\lambda^{k} I+\sum_{n=0}^{k-1} \lambda^{n} B_{n}, \quad B_{n} \in L,|\lambda| \leqslant 1,
$$

где $A_{+}(\lambda) \in H_{\alpha}^{+}(\Gamma, L)$, причем $A_{+}(\lambda)$ обратим при $\lambda \in G^{+} \cup \Gamma$, а $P_{k}(\lambda)$ обратим при $\lambda \in G^{-} \cup \Gamma$.

ЗАмечАниЕ 1. При выполнении условия (1) индекс $k$ функции $(A(\zeta) f, f)$, $|\zeta|=1$, определяющий количество ее корней в $G^{+}$, не зависит от выбора вектора $f \neq 0[2]$.

Доказательство утверждения (2) основано на следующей лемме.

Лемма 1 [1]. Пусть $\alpha \in(0,1)$ и оператор-функция $A(\zeta)$ принадлежит $H_{\alpha}(\Gamma, L)$. Если выполнено условие $(1)$ и $k=\operatorname{ind}_{\Gamma}(A(\zeta) f, f)(f \neq 0)$, то

$$
A(\zeta)=\zeta^{k} A_{+}(\zeta) A_{-}(\zeta), \quad \zeta \in \Gamma, A_{ \pm}(\zeta) \in H_{\alpha}^{ \pm}(\Gamma, L),
$$

причем $A_{+}(\lambda)\left(A_{-}(\lambda)\right)$ обратим при $\lambda \in G^{+} \cup \Gamma\left(\lambda \in G^{-} \cup \Gamma\right)$ u $A_{-}(\infty)=I$. 
Условие (1) имеет геометрический характер: оно формулируется в терминах выбранного скалярного произведения пространства $H$. Это условие равносильно следующему [2]: точка $\lambda=0$ для любого $\zeta \in \Gamma$ не принадлежит замыканию числового образа $W(A(\zeta))$ оператора $A(\zeta)$ :

$$
0 \notin \overline{W(A(\zeta))} \quad \forall \zeta \in \Gamma .
$$

В случае гильбертова пространства числовой образ оператора $A \in L$ является выпуклым множеством и $\sigma(A) \subset \overline{W(A)}$ [3]. Следовательно, выпуклая оболочка conv $\sigma(A)$ спектра оператора $A$ принадлежит замыканию его числового образа (множество conv $\sigma(A)$ является замкнутым в силу компактности множества $\sigma(A), A \in L)$. Таким образом, при выполнении условия (1) получаем, что выполняется условие

$$
0 \notin \operatorname{conv} \sigma(A(\zeta)) \quad \forall \zeta \in \Gamma .
$$

В данной статье показано, что при выполнении условия (4) для операторфункции $A(\zeta) \in C(\Gamma, L)$ возможно введение понятие индекса $k=\operatorname{ind}_{\Gamma} A(\zeta)$, и если, кроме того, $A(\zeta) \in H_{\alpha}^{+}(\Gamma, L)$, то справедлив аналог утверждения $(2)$, а именно, $A(\zeta)$ допускает факторизацию вида

$$
A(\lambda)=A_{1,+}(\lambda) P_{k}(\lambda) A_{2,+}(\lambda), \quad A_{j,+}(\lambda) \in H_{\alpha}^{+}(\Gamma, L), j=1,2,|\lambda| \leqslant 1,
$$

причем $A_{j,+}(\lambda)$ обратимы при $|\lambda| \leqslant 1$.

Отметим, что при фиксированном $\zeta_{0} \in \Gamma$ из (4) вытекает существование такого эквивалентного скалярного произведения $(\cdot, \cdot)_{0}$ в $H$, в котором для $A\left(\zeta_{0}\right)$ выполняется условие (3) (см. $[4,5])$. В общем случае для некоммутирующего семейства операторов $A(\zeta)$ из условия (4) не следует существования не зависящего от $\zeta \in \Gamma$ скалярного произведения, в котором бы выполнялось условие (3) для всех $\zeta \in \Gamma$. Соответствующий простой пример приведен в разд. 3. Этот же пример иллюстрирует точность утверждения (5), а именно, показывает, что условие (4) не гарантирует существования факторизации вида (2). Приведен также пример оператор-функции $A(\zeta)$, удовлетворяющей условию $(4)$, для которой условие (3) не выполняется ни в каком скалярном произведении, однако $A(\lambda)$ допускает факторизацию (2).

2. Теорема о факторизации. Далее понадобится следующее вспомогательное утверждение.

Лемма 2. Пусть оператор-функция $A(\zeta)$ принадлежит $H_{\alpha}(\Gamma, L), 0<\alpha \leqslant 1$, причем замкнутое ограниченное множество $\Sigma=\overline{\bigcup_{\zeta \in \Gamma} \sigma(A(\zeta))}$ лежит в открытой левой полуплоскости $\operatorname{Re} \lambda<0$. Тогда

$$
S(\zeta):=2 \int_{0}^{\infty} \exp \{A(\zeta) t\} \exp \left\{\left(A^{*}(\zeta)\right) t\right\} d t \in H_{\alpha}(\Gamma, L) .
$$

ДокАЗАТЕЛЬСтво. Выберем такой положительно ориентированный замкнутый жорданов контур $\gamma$, что $\operatorname{Re} \lambda \leqslant-r<0$ для любого $\lambda \in \gamma$ и $\Sigma$ лежит внутри $\gamma$. При этом для оператора $\exp \{A(\zeta) t\}$ справедливо представление [4]

$$
\exp \{A(\zeta) t\}=-\frac{1}{2 \pi i} \int_{\gamma} \exp \{\lambda t\} R(A(\zeta), \lambda) d \lambda \quad \forall \zeta \in \Gamma
$$

и выполняется оценка

$$
\|R(A(\zeta), \lambda)\| \leqslant c \quad \forall \zeta \in \Gamma, \lambda \in \gamma,
$$


которая вытекает из непрерывности оператор-функции $R(A(\zeta), \lambda)$ как функции двух переменных $\zeta \in \Gamma, \lambda \in \gamma$.

Из (7), (8) получаем оценку

$$
\|\exp \{A(\zeta) t\}\| \leqslant c_{1} \exp \{-r t\}, \quad t>0, \zeta \in \Gamma
$$

с постоянной $c_{1}=c l(\gamma) /(2 \pi)$, где $l(\gamma)$ - длина кривой $\gamma$. Далее, из (7) следует равенство

$$
\begin{aligned}
\exp \{ & \left.A\left(\zeta_{1}\right) t\right\}-\exp \left\{A\left(\zeta_{2}\right) t\right\} \\
& =-\frac{1}{2 \pi i} \int_{\gamma} \exp \{\lambda t\} R\left(A\left(\zeta_{1}\right), \lambda\right)\left[A\left(\zeta_{2}\right)-A\left(\zeta_{1}\right)\right] R\left(A\left(\zeta_{2}\right), \lambda\right) d \lambda,
\end{aligned}
$$

на основании которого, с учетом (9), заключаем, что при $t>0, \zeta \in \Gamma$ справедлива оценка

$$
\left\|\exp \left\{A\left(\zeta_{1}\right) t\right\}-\exp \left\{A\left(\zeta_{2}\right) t\right\}\right\| \leqslant c_{2} \exp \{-r t\}\left\|A\left(\zeta_{1}\right)-A\left(\zeta_{2}\right)\right\|
$$

с постоянной $c_{2}=c c_{1}$. Утверждение леммы вытекает из соотношения

$$
\begin{aligned}
S\left(\zeta_{1}\right)-S\left(\zeta_{2}\right)=2 & \int_{0}^{\infty} \exp \left\{A\left(\zeta_{1}\right) t\right\}\left[\exp \left\{A^{*}\left(\zeta_{1}\right) t\right\}-\exp \left\{A^{*}\left(\zeta_{2}\right) t\right\}\right] d t \\
& +2 \int_{0}^{\infty}\left[\exp \left\{A\left(\zeta_{1}\right) t\right\}-\exp \left\{A\left(\zeta_{2}\right) t\right\}\right] \exp \left\{A^{*}\left(\zeta_{2}\right) t\right\} d t
\end{aligned}
$$

и оценок $(9),(10)$, примененных к оператор-функциям $A(\zeta)$ и $A^{*}(\zeta)$.

ЛЕмма 3. Пусть оператор-функциия $A(\zeta)$ принадлежит $C(\Gamma, L)$. Предположим, что выполнено условие (4). Тогда найдется такая скалярная функция $a(\zeta) \in H_{1}(\Gamma)$, что для некоторого $r_{0}>0$ выполняется неравенство

$$
\operatorname{Re}(a(\zeta) \sigma(A(\zeta))) \leqslant-r_{0} \quad \forall \zeta \in \Gamma .
$$

ДокАЗАТЕЛьство. Из компактности окружности Г и свойства полунепрерывности сверху спектра оператора из $L$ (см. [3, с. 61]) нетрудно заключить, что найдется такое $r_{0}>0$, для которого

$$
\operatorname{dist}(\operatorname{conv} \sigma(A(\zeta)),\{0\}) \geqslant r_{0} \quad \forall \zeta \in \Gamma \text {. }
$$

При этом в силу выпуклости множества conv $\sigma(A(\zeta))$ для любого $\zeta \in \Gamma$ существует такое число $a_{\zeta} \in \mathbb{C}$, что справедливо неравенство

$$
\operatorname{Re}\left(a_{\zeta} \operatorname{conv} \sigma(A(\zeta))\right) \leqslant-r_{0}
$$

Отсюда легко выводится существование требуемой функции $a(\zeta)$ (например, используя компактность окружности $\Gamma$, можно построить функцию $a(\zeta) \in H_{1}(\Gamma)$ в виде кусочно линейной функции относительно переменного $\zeta \in \Gamma-$ см. построения леммы 3 работы [2]). Лемма доказана.

ЗАмЕЧАНИЕ 2. Пусть выполнены условия леммы 3 и $a_{0}(\zeta), a_{1}(\zeta)-$ произвольные непрерывные функции от $\zeta \in \Gamma$, для которых выполняется утверждение $(11)$. Тогда для любого $\varepsilon \in[0,1]$ непрерывная функция $a_{\varepsilon}(\zeta)=(1-\varepsilon) a_{0}(\zeta)+\varepsilon a_{1}(\zeta)$ не обращается в нуль при $\zeta \in \Gamma$. Таким образом, функции $a_{0}(\zeta), a_{1}(\zeta)$ являются гомотопными и поэтому имеют равные индексы (см. [6, гл. 1]): $\operatorname{ind}_{\Gamma} a_{0}(\zeta)=$ $\operatorname{ind}_{\Gamma} a_{1}(\zeta)$.

Лемма 3 и замечание 2 позволяют дать следующее определение. 
ОПРЕДЕЛЕНИЕ. Пусть Для оператор-функции $A(\zeta) \in C(\Gamma, L)$ выполнено условие (4). Индексом $\operatorname{ind}_{\Gamma} A(\zeta)$ на $\Gamma$ называется индекс функции $1 / a(\zeta)$ :

$$
\operatorname{ind}_{\Gamma} A(\zeta):=\operatorname{ind}_{\Gamma} 1 / a(\zeta)=-\operatorname{ind}_{\Gamma} a(\zeta),
$$

где $a(\zeta) \in C(\Gamma)-$ произвольная функция, для которой выполняется неравенство (11) с некоторым $r_{0}>0$.

Отметим, что данное определение тесно связано с понятием степени вращения многозначного отображения $\zeta \rightarrow \operatorname{conv} \sigma(A) \subset \mathbb{C}, \zeta \in \Gamma$ (см. [7]). Ясно, что при выполнении условия (1) справедливо равенство $\operatorname{ind}_{\Gamma} A(\zeta)=\operatorname{ind}_{\Gamma}(A(\zeta) f, f)$ для любого ненулевого вектора $f \in H$. Пусть $A(\zeta) \in C(\Gamma, L)$ удовлетворяет (4) и $\gamma-$ замкнутый контур из доказательства леммы 2. Тогда если $a(\zeta) \in$ $H_{1}(\Gamma)$ - функция из утверждения леммы 3 , то можно определить многозначную логарифмическую оператор-функцию

$$
\ln A(\zeta)=-\frac{1}{2 \pi i} \int_{\gamma}(\ln \lambda) R(a(\zeta) A(\zeta), \lambda) d \lambda-(\ln a(\zeta)) I, \quad \zeta=e^{i \varphi},
$$

где на $\gamma$ выбрана однозначная ветвь логарифма $\ln \lambda$. При этом $A(\zeta)=e^{\ln A(\zeta)}$, $\zeta \in \Gamma$, и, как и в скалярном случае, получаем для индекса $k=\operatorname{ind}_{\Gamma} A(\zeta)$ равенство

$$
k=-\operatorname{ind}_{\Gamma} a(\zeta)=\frac{1}{2 \pi i}\left[\ln A\left(e^{(\varphi+2 \pi) i}\right)-\ln A\left(e^{\varphi i}\right)\right], \quad \varphi \in \mathbb{R} .
$$

Аналогом леммы 1 в случае выполнения условия (4) является следующее утверждение.

Лемма 4. Пусть для оператор-функции $A(\zeta) \in H_{\alpha}(\Gamma, L), \alpha \in(0,1)$, вblполняется условие (4) $и$, в соответствии с определением (12), $k=\operatorname{ind}_{\Gamma} A(\zeta)$. Тогда $A(\zeta)$ допускает факторизацию

$$
A(\zeta)=\zeta^{k} A_{1,+}(\zeta) A_{-}(\zeta) A_{2,+}(\zeta)
$$

где $A_{j,+}(\zeta) \in H_{\alpha}^{+}(\Gamma, L), j=1,2, A_{-}(\zeta) \in H_{\alpha}^{-}(\Gamma, L)$, причем $A_{j,+}(\lambda)$ обратимь при $\lambda \in G^{+} \cup \Gamma$, а $A_{-}(\lambda)$ обратим при $\lambda \in G^{-} \cup \Gamma$ u $A_{-}(\infty)=I$.

ДокАЗАТЕЛЬСтво. Пусть $a(\zeta) \in H_{1}(\Gamma)-$ функция из утверждения леммы 3 . Тогда, согласно лемме 2 , самосопряженная оператор-функция

$$
S(\zeta)=2 \int_{0}^{\infty} \exp \{\widetilde{A}(\zeta) t\} \exp \left\{\widetilde{A}^{*}(\zeta) t\right\} d t, \quad \widetilde{A}(\zeta)=a(\zeta) A(\zeta),
$$

принадлежит $H_{\alpha}(\Gamma, L)$. При этом справедливы соотношения [4]

$$
\operatorname{Re}(\widetilde{A}(\zeta) S(\zeta))=-I, \quad(S(\zeta) f, f) \geqslant \frac{1}{2\|\widetilde{A}(\zeta)\|}, \quad \zeta \in \Gamma .
$$

В частности, согласно [8] (можно воспользоваться также леммой 1), операторфункция $S(\zeta)$ допускает факторизацию $S(\zeta)=S_{+}(\zeta) S_{-}(\zeta)$, где $S_{+}(\zeta) \in H_{\alpha}^{+}(\Gamma, L)$ $\left(S_{-}(\zeta) \in H_{\alpha}^{-}(\Gamma, L)\right)$, причем $S_{+}(\zeta)\left(S_{-}(\zeta)\right)$ обратим при $\zeta \in G^{+} \cup \Gamma\left(\zeta \in G^{-} \cup \Gamma\right)$ и $S_{-}(\infty)=I$. Тогда, применяя лемму $1 \mathrm{k} \widetilde{A}(\zeta) S(\zeta)$, получаем, что

$$
a(\zeta) A(\zeta) S_{+}(\zeta) S_{-}(\zeta)=M_{+}(\zeta) M_{-}(\zeta), \quad \zeta \in \Gamma, M_{ \pm}(\zeta) \in H_{\alpha}^{ \pm}(\Gamma, L),
$$

где $M_{+}(\zeta)\left(M_{-}(\zeta)\right)$ обратим внутри (вне) $\Gamma$ и $M_{-}(\infty)=I$. Далее, функция $1 / a(\zeta)$ допускает факторизацию $1 / a(\zeta)=\zeta^{k} a_{+}(\zeta) a_{-}(\zeta), a_{ \pm}(\zeta) \in H_{\alpha}^{ \pm}(\Gamma)$, причем 
$a_{+}(\zeta)\left(a_{-}(\zeta)\right)$ обратима при $\zeta \in G^{+} \cup \Gamma\left(\zeta \in G^{-} \cup \Gamma\right)$ и $a_{-}(\infty)=1$. Из (14) получаем факторизацию (13) с оператор-функциями

$$
A_{1,+}(\zeta)=a_{+}(\zeta) M_{+}(\zeta), \quad A_{-}(\zeta)=a_{-}(\zeta) M_{-}(\zeta) S_{-}^{-1}(\zeta), \quad A_{2,+}(\zeta)=S_{+}^{-1}(\zeta) .
$$

Лемма доказана.

Теорема. Пусть оператор-функциия $A(\zeta)$ принадлежит $H_{\alpha}^{+}(\Gamma, L), \alpha \in(0,1)$, $u$ для нее выполнено условие (4), причем $k=\operatorname{ind}_{\Gamma} A(\zeta)$. Тогда $k \geqslant 0 u A(\zeta)$ допускает факторизациню (5), где

$$
A_{j,+}(\lambda) \in H_{\alpha}^{+}(\Gamma, L), \quad j=1,2, \quad P_{k}(\lambda)=\lambda^{k} I+\sum_{n=0}^{k-1} \lambda^{n} B_{n}, \quad B_{n} \in L,
$$

причем $A_{j,+}(\lambda)$ обратим при $\lambda \in G^{+} \cup \Gamma$, а пучок операторов $P_{k}(\lambda)$ обратим nрu $\lambda \in G^{-} \cup \Gamma$.

ДокАЗАТЕЛЬство. По лемме 4 имеем факторизацию (13). В частности, имеет место равенство $A_{1,+}^{-1}(\zeta) A(\zeta) A_{2,+}^{-1}(\zeta)=P_{k}(\zeta), \zeta \in \Gamma$, где $P_{k}(\zeta)=\zeta^{k} A_{-}(\zeta)$. Так как по условию $A(\zeta) \in H_{\alpha}^{+}(\Gamma, L)$, то отсюда заключаем, что $P_{k}(\zeta)$ продолжается аналитическим образом на всю комплексную плоскость $\mathbb{C}$. С другой стороны, $A_{-}(\infty)=I$ и, значит, $k \geqslant 0$ и $P_{k}(\lambda)$ является полиномиальной оператор-функцией вида (15), причем $P_{k}(\lambda)$ обратим при $\lambda \in G^{-} \cup \Gamma$. Теорема доказана.

В условиях теоремы из факторизации (5) вытекает, что спектр операторфункции $A(\lambda)$ (т. е. множество тех $\lambda$, при которых $A(\lambda)$ не является непрерывно обратимым оператором), расположенный в круге $G^{+} \cup \Gamma$, совпадает со спектром пучка операторов $P_{k}(\lambda)$.

3. Примеры. В двумерном гильбертовом пространстве $H=\mathbb{C}^{2}$ рассмотрим голоморфную оператор-функцию

$$
A(\lambda)=\left(\begin{array}{cc}
2-4 \lambda & 2 \lambda^{2}-2 \lambda+1 \\
-2 & 0
\end{array}\right), \quad \lambda \in \mathbb{C} .
$$

Вычисления показывают, что собственными значениями оператора $A(\zeta)$ являются числа $\mu_{ \pm}(\zeta)=-2 \zeta+1 \pm i$ и множество conv $\sigma(A(\zeta))$ образует отрезок, соединяющий точки $\mu_{+}$и $\mu_{-}$. При этом для функции $a(\zeta)=1 / \zeta$ выполняется равенство

$$
\operatorname{Re}\left\{a(\zeta) \mu_{ \pm}(\zeta)\right\}=\operatorname{Re}\left\{-2+(1 \pm i) \zeta^{-1}\right\} \leqslant-2+\sqrt{2}<0, \quad|\zeta|=1 .
$$

Оценка (17) показывает, что $\operatorname{ind}_{\Gamma} A(\zeta)=\operatorname{ind}_{\Gamma} \zeta=1$ и $A(\zeta)$ удовлетворяет условиям теоремы с $k=1$. Спектр оператор-функции $A(\lambda)$ состоит из простых собственных значений $\lambda=(1 \pm i) / 2$, которые лежат в единичном круге. При этом каждому из этих собственных значений отвечает один и тот же собственный вектор $\vec{e}=\{0,1\}^{T}$ (индекс $T$ означает транспонирование). Таким образом, система нормированных собственных векторов оператор-функции $A(\lambda),|\lambda|<1$, не образует базиса в пространстве $\mathbb{C}^{2}$. Так как в конечномерном случае из факторизации (2) с $k=1$ вытекает, что такая система должна образовывать базис, то приведенный пример показывает, что условие (4) не эквивалентно условию (1): не существует никакого скалярного произведения $(\cdot, \cdot)$ в $\mathbb{C}^{2}$, при котором для 
$A(\lambda)$ из (16) выполняется условие (1). При этом $A(\lambda)$ допускает факторизацию вида (5) с оператор-функциями $A_{1,+}(\lambda)=I$ и

$$
A_{2,+}(\lambda)=\left(\begin{array}{cc}
-4 & 2 \lambda-1 \\
0 & -1
\end{array}\right), \quad P_{1}(\lambda)=\left(\begin{array}{cc}
\lambda-1 / 2 & -1 / 2 \\
1 / 2 & \lambda-1 / 2
\end{array}\right) .
$$

Отметим, что собственным значениям $\lambda_{1}=(1-i) / 2, \lambda_{2}=(1+i) / 2$ линейного пучка операторов $P_{1}(\lambda)$ из (18) отвечают собственные векторы $\vec{e}_{1}=\{-i, 1\}^{T}$, $\vec{e}_{2}=\{i, 1\}^{T}$.

Из приведенного примера следует, что условие (1) не выполняется ни при каком скалярном произведении и для транспонированной к $A(\lambda)$ оператор-функции

$$
A^{T}(\lambda)=\left(\begin{array}{cc}
2-4 \lambda & -2 \\
2 \lambda^{2}-2 \lambda+1 & 0
\end{array}\right) .
$$

С другой стороны, переходя в (5), (18) к транспонированным матрицам, получаем для $A^{T}(\lambda)$ факторизацию $A^{T}(\lambda)=A_{2,+}^{T}(\lambda) P_{1}^{T}(\lambda)$. Таким образом, факторизация вида $(2)$ для оператор-функции из $H_{\alpha}^{+}(\Gamma, L)$ возможна и в случае, когда выполняется лишь условие (4) и не выполняется (1).

\section{ЛИТЕРАТУРА}

1. Маркус A. С., Мацаев В. И. К спектральной теории голоморфных оператор-функций в гильбертовом пространстве. Функц. анализ и его прил., 9, вып. 1, 76-77 (1975).

2. Маркус A. С., Мацаев В. И. О спектральных свойствах голоморфных операторфункций в гильбертовом пространстве. Матем. исследования, 9, вып. 4, 79-90 (1974).

3. Халмош П. Гильбертово пространство в задачах. Мир, М., 1970.

4. Далецккий Ю. Л., Крейн М. Г. Устойчивость решений дифференциальных уравнений в банаховом пространстве. Наука, М., 1970.

5. Гомилко А. М., Радзиевский Г. В. О числовых образах семейства коммутирующих операторов. Матем. заметки, 62, вып. 5, 787-791 (1997).

6. Красносельский М. А. Векторные поля на плоскости. ГИФМЛ, М., 1963.

7. Борисович Ю. Г., Гельман Б. Д., Мышкис А. Д., Обуховский В. В. Топологические методы в теории неподвижных точек многозначных отображений. УМН, 35, вып. 1, 59-126 (1980).

8. Gohberg I., Laiterer Yu. The factorization of operator-functions relative to a contour. II. The canonical factorization of operator-functions that are close to the identity operator. Math. Nachr., 54, 41-74 (1972).

Институт гидромеханики

НАН Украины, Киев

Поступило в редакцию e-mail: amg@ihm.kiev.ua 25 марта 2002 г. 